\title{
Seroprevalence of rubella-specific IgM and IgG antibodies among pregnant women in a Catholic Hospital in Yaoundé, Cameroon
}

\author{
Anna Aret ENO ${ }^{1}$, Louise Stéphanie MAKEMGUE ${ }^{1 *}$, Daves Stéphane TONYE III ${ }^{1}$, \\ Cheikh Saad Bouh BOYE ${ }^{2}$, Abdoulaye $\mathrm{SECK}^{3}$, Raymond BERCION ${ }^{3}$, \\ Christelle VAULOUP FELLOUS ${ }^{4}$ and Louis Albert TCHUEM TCHUENTE ${ }^{1}$
}

\author{
${ }^{1}$ Department of Animal Biology, Faculty of Science, University of Yaounde I. BP 812, Yaounde, Cameroon. \\ ${ }^{2}$ Department of Microbiology, Faculty of Science, Cheikh Anta Diop University. BP 45515 Dakar Fann, \\ Senegal. \\ ${ }^{3}$ Biology and Medical Analysis Laboratory of Pasteur Institut, Dakar, Senegal. . BP 220, Dakar, Senegal. \\ ${ }^{4}$ AP-HP, Paul Brousse Hospital, Virology-CNR Maternofoetal rubella infections, University of Paris-Sud, \\ UMR-S 785. Hôpital Paul Brousse. 12, avenue Paul Vaillant-Couturier. 94800 Villejuif, \\ Tel : +33145593721/ Fax: +33145593724 \\ *Corresponding author; E-mail: stephaniemakemgue@gmail.com; Tel: +237679649388
}

\section{ACKNOWLEDGMENTS}

The present study was financially supported by the Ministry of Foreign Affairs and the Service of French Cultural Cooperation and Action in Cameroon.

\begin{abstract}
Rubella virus (RV) infection is responsible of an unresolved clinical complication that affects newborns and children. During the first trimester of pregnancy, it often causes severe birth defects known as congenital rubella syndrome (CRS). This study reports the seroprevalence of the RV-specific IgM and IgG antibodies, its relationship with the duration of pregnancy and past history of abortion in pregnant women at Yaoundé in order to help strategies to eliminate rubella and to prevent Congenital Rubella Syndrome (CRS) in Cameroon. Four Hundred (400) pregnant women were screened for rubella immunoglobulins G ( $\operatorname{IgG}$ ) and M (IgM), using the chemiluminescent microparticle immunoassay for the detection of IgG and IgM antibodies on the ARCHITECT $i$ system at the Laboratory of Medical Analysis of Pasteur Institute, Senegal. Out of the 400 pregnant women tested, $367(91.75 \%)$ were positive for RV-IgG while only $5(1.25 \%)$ were positive for RVIgM. A higher number of pregnant women in the first trimester of pregnancy tested positive for $\operatorname{IgG}(91.8 \%)$. None of the possible risk factors were significantly associated with infection. The presence of rubella RV-IgM and RV-IgG in pregnant women predisposes babies to CRS and emphasizes on the initiation of a vaccination policy of those who are susceptible in Cameroon.

(C) 2018 International Formulae Group. All rights reserved.
\end{abstract}

Keywords: Antibodies, Cameroon, pregnant women, rubella, seroprevalence.

\section{INTRODUCTION}

Rubella is an infection caused by an enveloped positive-stranded ribonucleic acid virus of the genus Rubivirus of the Togavirus family (Murray, 2006.). Rubella is characterized by fever and rashes and it easily 
moves from one person to another through respiratory secretions. In post-natal RV infection, the virus causes lymphadenopathy, and a short-lived morbilliform rash in addition to mild rash-like and low-grade fever (Brooks, 2013). Its public health importance arises from the ability of the rubella virus (RV) to infect the fetus and cause severe birth defect. Indeed, among infants born from mothers infected with RV during the first trimester of pregnancy, 75 to $90 \%$ have congenital defects mostly affecting the brain, heart, eyes and ears (Andrade et al., 2006; Reef et al., 2011; Agbede, 2011). Rubella infections are prevented by active immunization program using live, attenuated virus vaccine. The vaccine is combined with measles and mumps vaccine. Considering the fact that Cameroon is one of the countries that has not implemented a routine vaccination scheme, an eventual outbreak cannot be over emphasized. There is a need to know the epidemiology of rubella in pregnant women because of the congenital rubella syndrome (CRS), and how many primiparous infections in the first trimester of pregnancy may occur. During the course of a primary infection, RV- IgM appears first, with its peak between the $7^{\text {th }}$ $10^{\text {th }}$ day after infection and usually becomes negative 6 to 12 weeks later. Secondly, the $\mathrm{RV}$-IgG become positive and usually persists throughout life indicating that, the patient is immune. In countries where rubella is endemic, RV-IgM usually indicates a recent infection. If RV-IgM is absent but RV-IgG is positive, it indicates an old infection and immunity (Lombardo, 2012). Most publications on the incidence and prevalence of rubella infection in pregnant women conducted in Cameroon have been performed using RV-IgG, but not in association with IgM (Fokunang et al., 2010; Nimpa et al., 2017).

The purpose of this study was to detect the presence of both RV-IgM and RV-IgG antibodies in pregnant women attending Catholic Hospital, thereby giving a complete picture of the occurrence of the disease among pregnant women in Yaoundé, Cameroon in order to help strategies to eliminate rubella and to prevent Congenital Rubella Syndrome 'CRS) in Cameroon.

\section{MATERIALS AND METHODS}

The study was carried out on a cohort group of 400 pregnant women attending Antenatal clinics (Catholic Hospital in Yaounde). Ethical approval was obtained from the National Ethical Committee. A consent form was signed by each participant.

A structured questionnaire was designed and standardised. Data was collected through structured interviews. Three to four millilitres of blood were collected from the subjects by Venepuncture into labelled sterile sample tubes and allowed to clot undisturbed at room temperature. Sera were separated by centrifugation at 5,000 revolutions per minute (rpm) for 15 minutes and stored in 3 serum vial aliquots at $-20^{\circ} \mathrm{C}$ until analyses.

Sera were tested for RV-IgM, and RVIgG, using ARCHITEC System Ci4100 (Abbott Diagnostics).The assays were carried out according to the manufacturer's instructions. RV-IgG were interpreted as positive if titers were above $10 \mathrm{IU} / \mathrm{ml}$ and interpreted as negative if titers were below or equal to $4.9 \mathrm{IU} / \mathrm{ml}$. An IgG value between 5.0 and 9.9 was considered as borderline and women were considered as susceptible if they were negative. Concerning RV-IgM, samples were interpreted as positive if index was above to 1.60 and interpreted as negative if index was below or equal to 1.20. An IgM value between 1.20 and 1.60 was considered as borderline.

Data entry was with the SAS version 9.1 (Statistical Analyses system, USA). Data was statistically tested at a critical level for statistical significance of $95 \%$ ( $\mathrm{p}=0.05$ ) using the Chi-square and 95\% Confidence Interval.

\section{RESULTS \\ Characteristics of the population studied}

Most patients were in the age group of $23-27$ years $(140 / 400,35.00 \%)$, followed by the age group of 18-22 years $(139 / 400$, $34.75 \%)$, and $4.25 \%(17 / 400)$ of the patients were aged between 13 and 17 years (Figure 1). The mean maternal age of the participants was 24 years old. All the patients had never 
been vaccinated against rubella, and the vaccination state was unknown in about $94 \%$ of the cases.

A total of $337(84.25 \%)$ pregnant women were from urban areas and 257 $(64.25 \%)$ of all pregnant women were workers. One hundred and twenty two $(30.5 \%)$ of the women were in their first trimester of pregnancy and $269(67.25 \%)$ were in their second trimester of pregnancy. Only fifty nine $(14.75 \%)$ of the women were married. Of the 400 pregnant women who participated in the study, $284(71.0 \%)$ were multigravid, $95(23.75 \%)$ had history of previous pregnancy losses while $276(69.0 \%)$ had living children. Only $20.75 \%$ and $31 \%$ of the women were symptomatic with fever and rash respectively. Additionally, reproductive characteristics such as the number of stillbirths, spontaneous abortions and surviving children, were reported (Table 1).

Some of the common clinical symptoms associated with rubella virus infection were observed in the pregnant women.

\section{Prevalence of IgG and IgM rubella antibodies}

Of the 400 women, 367 (91.75\%) were tested positive for RV-IgG. A total of 18 women $(4.5 \%)$ had negative RV-IgG, thus being at high risk of contracting rubella infection during pregnancy (Figure2). Out of the $367(91.75 \%)$ pregnant women who were positive for RV-IgG, 5 (1.36\%) were also positive for RV-IgM, indicating an acute rubella infection (Figure 3). All of the remaining $18(4.5 \%)$ pregnant women were negative for both RV-IgG and RV-IgM. Among the 95 pregnant women who had lost previous pregnancies, all of them were positive for RV-IgG and 5 had a borderline RV-IgG result. Borderline RV-IgG samples were also negative for RV-IgM. A higher number of pregnant women in the first and second trimester were tested positive for RVIgG $(91.8 \%$ and $92.1 \%$ respectively) compared to those in their third trimester $(77.8 \%)$. However, this difference was not statistically significant $(\mathrm{p}=0.628)$.

\section{Risk factors for RV infection}

All the possible risk factors considered in this study such as type of occupation, area of residence and number of living children in the house were not significant. Additionally, analysis (IgM and $\operatorname{IgG}$ ) showed that age, trimester of pregnancy and socio-demographic data were not significant risk factors for rubella virus infection as shown in Tables 2 to 4.

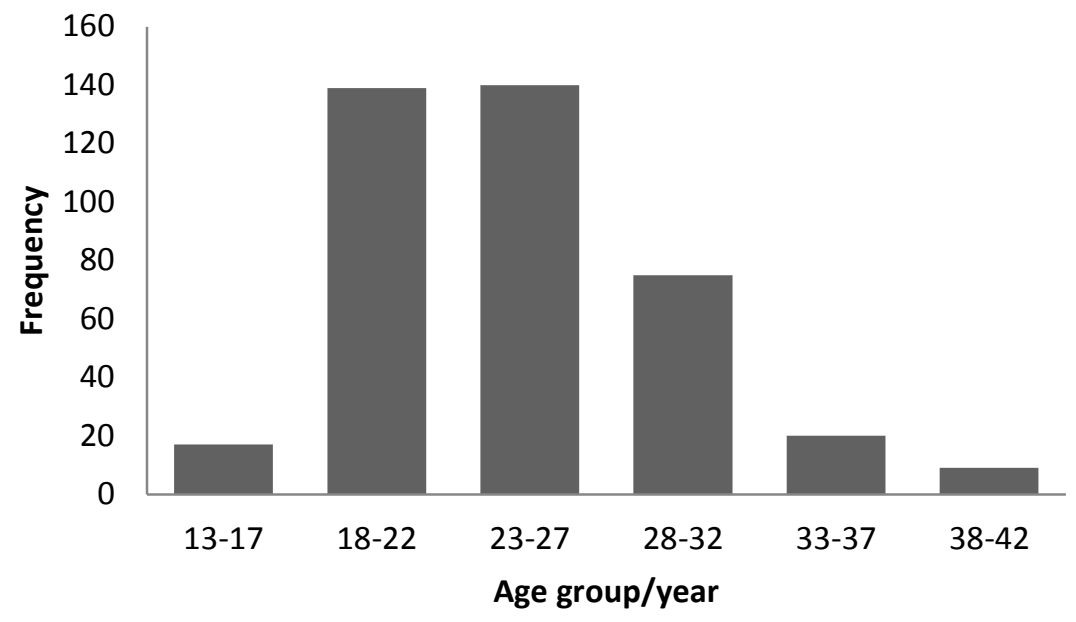

Figure 1: Distribution of subjects by age groups. 
Table 1: Distribution of subjects according to stillbirths, spontaneous abortions and surviving children.

\begin{tabular}{lcc}
\hline Indicators & Frequency & Percentage \\
\hline Stillbirths & & \\
None & 378 & 94.5 \\
$\geq 1$ & 22 & 5.5 \\
Total & 400 & 100.0 \\
Spontaneous abortions & & \\
None & 305 & 76.25 \\
$\geq 1$ & 95 & 23.75 \\
Total & 400 & 100.0 \\
Surviving children & & \\
None & 135 & 33.75 \\
$\geq 1$ & 265 & 66.25 \\
Total & 400 & 100.0 \\
& & \\
\hline
\end{tabular}

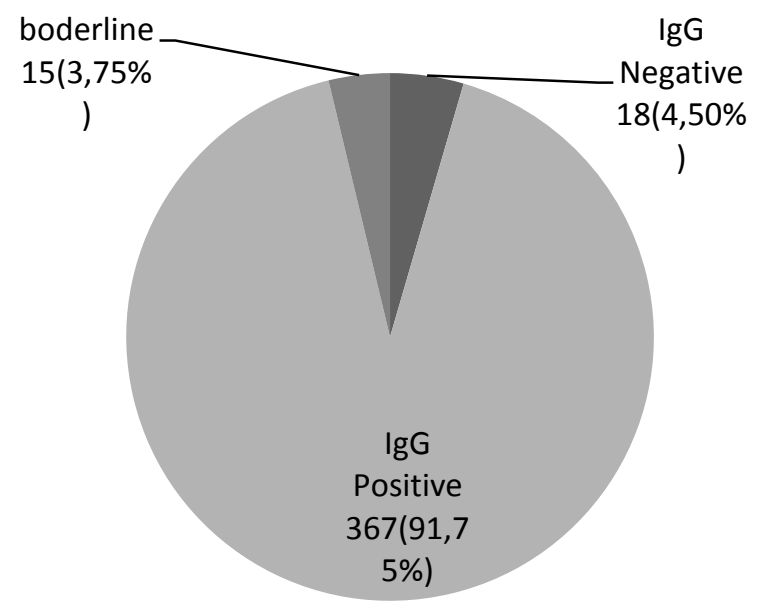

Figure 2: RV-IgG results.

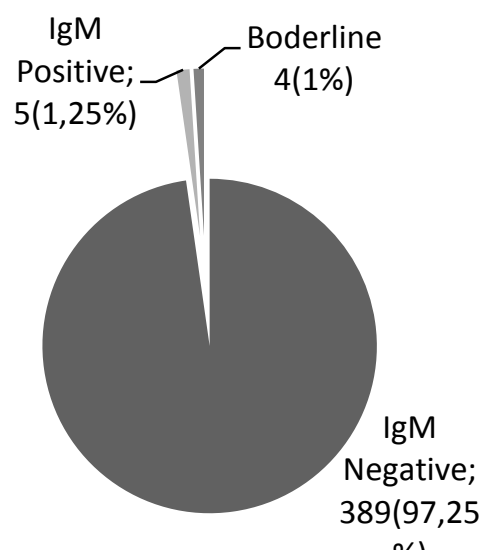

$\%)$

Figure 3: RV-IgM results. 
Table 2: Age distribution of pregnant women with $\operatorname{IgM}$ and $\operatorname{IgG}$ antibodies.

\begin{tabular}{|c|c|c|c|c|c|c|c|}
\hline \multirow{2}{*}{$\begin{array}{c}\text { Age } \\
\text { groups } \\
\text { (year) }\end{array}$} & \multirow[t]{2}{*}{ Numberanalyzed } & \multicolumn{3}{|c|}{ RubellaIgM } & \multicolumn{3}{|c|}{ RubellaIgG } \\
\hline & & $\begin{array}{l}\text { Number } \\
\text { positive }\end{array}$ & percentage & P-value & $\begin{array}{l}\text { Number } \\
\text { positive }\end{array}$ & percentage & $\begin{array}{l}\text { P-value } \\
\end{array}$ \\
\hline $13-17$ & 17 & 0 & 0.00 & & 15 & 88.24 & \\
\hline $18-22$ & 139 & 3 & 2.16 & & 124 & 89.21 & \\
\hline $23-27$ & 140 & 1 & 0.71 & 0.075 & 131 & 93.57 & 0.733 \\
\hline $28-32$ & 75 & 1 & 1.33 & & 69 & 92.00 & \\
\hline $33-37$ & 20 & 0 & 0.00 & & 19 & 95.00 & \\
\hline $38-42$ & 9 & 0 & 0.00 & & 9 & 100.00 & \\
\hline
\end{tabular}

Table 3: Seroprevalence of rubella $\operatorname{IgM}$ and $\operatorname{IgG}$ antibodies among pregnant women according to their sociodemographic data.

\begin{tabular}{llllcccc}
\hline $\begin{array}{l}\text { Sociodemographic } \\
\text { data }\end{array}$ & Numberanalyzed & \multicolumn{3}{c}{ RubellaIgM } & \multicolumn{3}{c}{ RubellaIgG } \\
\cline { 3 - 8 } & & $\begin{array}{l}\text { Number } \\
\text { positive }\end{array}$ & percentage & P-value & $\begin{array}{c}\text { Number } \\
\text { positive }\end{array}$ & percentage & P-value \\
\hline Marital status & & 1 & 1.69 & 0.670 & 55 & 93.22 & 0.460 \\
$\begin{array}{l}\text { Married } \\
\text { Single }\end{array}$ & 59 & 4 & 1.17 & & 312 & 91.50 & \\
Educationalstatus & 341 & & & & & & \\
Primary & 24 & 0 & 0.00 & & 23 & 95.83 & 0.742 \\
Secondary & 333 & 4 & 1.20 & 0.35 & 303 & 90.99 & \\
Tertiary & 43 & 1 & 2.33 & & 41 & 95.35 & \\
Occupation & & & & & & & \\
$\quad$ Workers and student & 257 & 3 & 1.17 & & 236 & 91.8 & 0.267 \\
Housewives & 139 & 2 & 1.44 & 0.97 & 128 & 92.1 & \\
Health-care worker & 4 & 0 & 0.00 & & 3 & 75.00 & \\
\hline
\end{tabular}

Table 4: Seroprevalence of rubella IgM and IgG antibodies among pregnant women according to their trimester of pregnancy.

\begin{tabular}{lccccccc}
\hline $\begin{array}{l}\text { Trimesters } \\
\text { of pregnancy }\end{array}$ & Numberanalyzed & \multicolumn{3}{c}{ RubellaIgM } & \multicolumn{2}{c}{ RubellaIgG } \\
\cline { 3 - 8 } & & $\begin{array}{c}\text { Number } \\
\text { positive }\end{array}$ & percentage & $\begin{array}{r}\text { P-value } \\
\text { Number } \\
\text { positive }\end{array}$ & percentage & P-value \\
\hline First & 122 & 1 & 0.82 & & 112 & 91.80 & \\
Second & 269 & 4 & 1.49 & 0.881 & 248 & 92.19 & 0.628 \\
Third & 9 & 0 & 0.00 & & 7 & 77.78 & \\
Total & 400 & 5 & 1.25 & & 367 & 91.75 & \\
\hline
\end{tabular}




\section{DISCUSSION}

A high seroprevalence for RV among pregnant women was found in Yaounde. For those women who were induced during their first trimester of pregnancy, the presence of $\mathrm{RV}$-IgG is most likely due to an old rubella infection because RV-IgM were negative for them. However, for those induced later in pregnancy, in the absence of IgM, an earlier exposure during pregnancy cannot be excluded. This study has shown that rubella is quite common and active among the population including pregnant women as $1.36 \%$ of our patients had positive RV-IgM during pregnancy. Only $20.75 \%$ and $31 \%$ of the women were symptomatic with fever and rash respectively. This was probably due to the mild nature of the infection which could easily be confused with the malaria fever which is endemic in Cameroon. In addition, rubella-induced rashes are often misdiagnosed in developing countries such as Cameroon.

Our findings are consistent with results reported by Okikiola et al. (2015) as we do not report that seroprevalence of rubella increases with age. This is contrary to another study in Yaounde (Cameroon) in pregnant women (Fokunang et al., 2010) that reported increase with age. This difference was probably due to the high endemicity of the virus in Yaounde as it is in constant circulation.

The current study indicates that a significant number of pregnant women in Cameroon are at risk of acquiring RV infection. All women, including the infected patients, said that they had been never administered prophylactic vaccination. Antenatal health-talks in Cameroon routinely do not incorporate information on Rubella infection. Vaccination against rubella is also not part of the Cameroon national or local immunization programs (Fokunang et al., 2010). Preconception counselling of women of reproductive age about rubella is also not routine in Cameroon. There is probably a lack of awareness concerning rubella among the population but this should be investigated in specific studies.
Despite the fact that the majority of the pregnant women were educated up to the secondary and the tertiary level, the level of awareness and knowledge of rubella was very low among the women included in our study. This raises a serious problem, as it is obvious that knowledge of rubella do not prevent infection. This emphasizes that vaccination is the best means of prevention, and enlightenment without vaccination will achieve nothing.

A seroprevalence of $1.25 \%$ was obtained in our population for RV-IgM. All these pregnant women also had IgG antibody, suggesting either reinfection without consequences in foetus, or recent primary infection for those who were in first trimester of pregnancy with high risk of serious damage for their foetus. Most of these women were in their second and third trimesters of pregnancy, suggesting that they were infected earlier in pregnancy, as virtually all infected persons should have developed IgG antibodies by 30 days of post-infection (ClinLab Navigator Rubella, 2013). The $1.25 \%$ prevalence obtained is much higher than any that has been reported in Tanzania $(0.3 \%)$ but is less than any that has been reported in two studies in Nigeria (9\%, and 38.8\%) (Mwambe et al., 2014; Onakewhor and Chiwuzie, 2011; Okikiola et al., 2015). The high prevalence report in Tanzania obtained suggests the occurrence of an outbreak during the time of the study that might have gone unnoticed, as outbreaks of rubella may not always be recognized in developing countries such as Cameroon, and rubella-induced rashes are often misdiagnosed.

Antibodies were found in all the trimesters of pregnancy, with the highest prevalence $(92.1 \%)$ being in the second trimester. This agrees with the work of Okikiola et al. (2015) which showed the prevalence of $96.3 \%$. The highest prevalence observed in the second trimester may have been because most of the pregnant women were in their fourth and fifth months of pregnancy.

None of the characteristics in our study considered to be risk factors for RV infection 
were indeed statistically predisposing factor to $\mathrm{RV}$ infection. Some of the common clinical symptoms associated with rubella virus infection were observed in the pregnant women. The pregnant women made complaints mostly of fever and rash. Very few of these women were, however, positive for rubella infection (IgM antibody), suggesting that the fever and rash were due to other factors. This result shows that most of the infected patients were asymptomatic, and none of the clinical symptoms was significantly associated with the risk of infection.

There were possible cross-reactions of rheumatoid IgG complexes mimicking IgM antibody. Due to logistic constraints, we were unable to exclude other sources of potential IgM cross-reacting antibodies such as CMV, EBV, Toxoplasma, and Parvovirus infections. However, the presence of rubella-specific IgM or a significant rise in rubella-specific $\mathrm{IgG}$ could be indicative of recent infection (Hobman et al., 2007). Unfortunately, there are no medications for mothers with active infections. Therefore, routine antenatal testing for rubella antibody is a good practice irrespective of a woman's seronegative status in a previous pregnancy (Hobman et al., 2007).

\section{Conclusion}

We found a high seroprevalence of rubella $\operatorname{IgG}$ antibodies among pregnant women in Yaoundé. Up to $1.25 \%$ of fetuses of pregnant women in Yaounde are predisposed to CRS due to the presence of IgM and IgG antibodies. The immunity gap in this study was high and this therefore buttressed the need for rubella vaccination to be given to women who are seronegative and their children. However, further studies on the susceptibility of women of child bearing age needs to be carried out countrywide. Furthermore, studies to determine the prevalence of CRS are also needed.

\section{COMPETING INTERESTS}

During the study, many of the pregnant women at the antenatal clinic were in the second trimester, making it difficult to adequately assess the risk burdens associated with infection of rubella virus. Also, the majority of the pregnant women did not want to take part in the study, saying it was not necessary and they did not need it. This made sample collection very difficult.

\section{ACKNOWLEDGMENTS}

We thank the nurses and lab technicians at the antenatal clinics of the health centres (both of Cameroon and Senegal) that participated in our study; the patients too. The authors are grateful to the Department of Animal Biology and Physiology, Faculty of Science, University of Yaounde, for its contribution to the study.

\section{REFERENCES}

Agbede OO, Adeyemi OO, Olatinwo AWO, SalisuTJ, Kolawole OM. 2011. SeroPrevalence of Antenatal Rubella in UITH.The. Open. Public. Health Journal., 4: 10-6.

Andrade JQ, Bunduki V, Curti SP, Figueiredo CA, de Olivera MS, Zugaib M. 2006. Rubella in pregnancy: Intrauterine transmission and perinatal outcome during a Brazilian epidemic. $J$. Clin.Virol., 35: 285-291.

Bamgboye AE, Afolabi KA, Esumeh FI and Enweani I B. 2004. Prevalence of rubella antibody in pregnant women in Ibadan, Nigeria. West. Afr. J. Med., 23(3): 245248.

Brooks GF, Carroll KC, Butel JN, Morse SA, Mietzer TA. 2013. Rubella (German Measles) Virus. In Medical Microbiology (26th edn), Jawetz, Melnick, Adelberg's (eds). McGraw-Hill Lange Companies: U.S.A., 4; 607-612.

ClinLab Navigator. Rubella. 2013. Available from: $\quad$ http://www.clinlab navigator.com/rubella.html.

Fokunang CN, Chia J, Ndumbe P, Mbu P, Atashili J. 2010. Clinical studies on seroprevalence of rubella virus in 
pregnant women of Cameroon regions. Afr. J. Clin. Exp. Microbiol., 11(2): 7994.

Hobman T, Chantler J. Knipe DM, Howley PM, Griffin DE, Martin, 2007. Rubella Virus and Fields Virology, PA, USA (5th edn). Lippincott Williams \& Wilkins Publishers; 1069-1100.

Lombardo PC. 2011. Dermatological manifestations of rubella. Available from:

http://www.emedicine.medscape.com/art icle/1133108-overview. Accessed March 22, 2012.

Murray M. 2006. The Rubella Virus. Microbiology and Immunology (3rd edn). Oxford University Press; 499-502.

Mwambe B, Mirambo MM, Mshana SE, Massinde AN, Kidenya BR, Denna M, Morona D, Majinge G and Uwe G. 2014. Sero-positivity rate of rubella and associated factors among pregnant women attending antenatal care in Mwanza, Tanzania. BMC. Pregnancy and Childbirth., 14: 95-99. DOI: http://www.biomedcentral.com/14712393/14/95
Nimpa MM, Ndze VN, Baonga F, Kobela M, Shey CW. 2017. Epidemiology of rubella infection in Cameroon: a 7-year experience of measles and rubella casebased surveillance, 2008-2014. British Medical Journal Open., 7: 1136-2016. DOI: 10.1136/ bmjopen-2016-012959

Okikiola MO, Aminu M, Randawa AJ, Adejo D S. 2015. Seroprevalence of rubellaspecific IgM and $\operatorname{IgG}$ antibodies among pregnant women seen in a tertiary hospital in Nigeria. International Journal of Women's Health, 7: 775-783. http://dx.doi.org/10.2147/IJWH.S68667

Onakewhor JU, Chiwuzie J. 2011. Seroprevalence survey of rubella infection in pregnancy at the University of Benin Teaching Hospital, Benin City, Nigeria. Niger. J. Clin. Pract., 14(2): 140 - 145. DOI: 10.4103/1119-3077

Reef SE, Strebel P, Dabbagh A, Gacic-Dobo M, Cochi S. 2011. Progress toward control of rubella and prevention of congenital rubella syndrome Worldwide. J. Infect. Dis., 204: 24-27. 